\title{
Does prior knowledge facilitate the detection of visual targets in random noise?
}

\author{
JOSEPH S. LAPPIN \\ Vanderbilt University, Nashville, Tennessee 37240 \\ and \\ WILLIAM R. UTTAL \\ University of Michigan, Ann Arbor, Michigan 48104
}

\begin{abstract}
The detectability of forms embedded within random visual noise has been found to be predictable from the autocorrelation transform of the stimulus pattern (Uttal, 1975). A basic assumption in the autocorrelation theory of form detection is that detectability is determined by the organization of the stimulus pattern, irrespective of the observer's prior knowledge or expectations about the characteristics of the form. This assumption was tested by determining the effect of the size of the set of alternative target forms on performance of a forced-choice detection task. The targets were composed of dots in a straight line, appearing in one of a specified set of 2,4 , or 8 alternative positions in a pattern of randomly distributed masking dots. Detection accuracy was found to decrease as set size increased, but this decrease was close to what was predicted on the assumption that the random background was independently confusable with the target at each of the alternative positions. Thus, prior knowledge of the set of alternative targets appeared to have no effect on the visual process, but only on the decision process by virtue of the features that were relevant criteria for deciding which of the two patterns on each trial was most likely to contain the target. This result is consistent with the autocorrelation theory. This experiment may illustrate how the decision process has influenced the performance in many other experiments that have been assumed to demonstrate an effect of prior knowledge on perception.
\end{abstract}

A significant development in contemporary research on visual form perception is the use of complex spatial and temporal patterns that are generated and displayed under control by a computer. The concept of "form" or "pattern" can thus be defined by the relational organization of a large number of identical elements.

This technique is illustrated in Uttal's (1975) research program on the detection of simple forms embedded in random patterns of dots that serve to mask or camouflage the form. Both target form (a deterministically defined pattern) and noise (a statistically defined masking pattern) are patterns of dots displayed on a cathode ray tube-the target being regular and periodic in some way, and the noise being random with uniform distribution in

Reprint request should be addressed to Joseph S. Lappin, Department of Psychology, Vanderbilt University, Nashville, Tennessee 37240 . We would like to express our appreciation to Ms. Thelma Eskin, who helped greatly in the running of the experiment and in the data analysis, and to V. R. Carlson and Sandra McNabb for their helpful comments on earlier drafts. This research was carried out in the Ann Arbor laboratory and was supported by NIMH Grant MH 24016-03, by NIMH Research Scientist Award MH 29941-05 to W. Uttal, and by NSF Grant BMS-75-19103 and a faculty travel grant from Vanderbilt University to J. Lappin. the plane. The general result obtained with this procedure is that detectability depends on the pattern of relationships among the components of the form rather than on the nature of the components (the dots) themselves. Specifically, the relative detectability of a large class of forms is well described by the autocorrelation function of the pattern (see Uttal, 1975, for a detailed account of the theory and empirical findings). This mathematical transform measures the periodicity or repetitiveness of the pattern, as well as certain properties of general organization.

An important characteristic of the autocorrelation theory is that detectability of a form is supposed to depend entirely on its spatial structure relative to that of the random noise background, irrespective of the observer's prior knowledge about the features of the form. Since this strong hypothesis is central to the current version of the autocorrelation theory, it merits a test; and that was the purpose of this experiment.

The observer's prior knowledge about the features of the target form was manipulated by varying the size of the set of alternative target forms that were presented in a forced-choice detection task. A larger number of alternative forms involves a larger number of potentially relevant features that 
must be analyzed by the detection process. Presumably, prior knowledge about the relevant features should be most beneficial when the alternative forms are perceptually distinct, so that the distinguishing features may be selectively attended and processed. In order to maximize the opportunity for selective processing of some features or forms at the expense of others, the alternative forms differed with respect to both spatial position and orientation, as shown in Figure 1. Because of the distinct differences between these forms, variation in set size might be expected to produce a larger effect on detectability for these forms than for many other sets of forms differing on other geometric features.

Previous research, however, provides conflicting indications about the effects of spatial uncertainty on form perception. Common assumptions about perceptual information-processing would suggest that information from separate spatial positions can often be separately attended and processed, due to the clear phenomenological and sensory differences between such stimuli. Evidence about the spatial and temporal characteristics of such a hypothesized attentional focus has been provided by a series of experiments by Eriksen and his colleagues (e.g., Colegate, Hoffman, \& Eriksen, 1973; Eriksen \& Hoffman, 1972, 1973; Hoffman, 1975). Using a preceding indicator to designate the position at which a target letter will subsequently appear, they have found that the RT for identifying the target letter is maximally facilitated by providing the information about spatial position approximately $200-300 \mathrm{msec}$ in advance of the target presentation, with the facilitation diminishing as the interval between spatial indicator and target letter is decreased. The spatial resolution of the hypothetical attentional focus seems to be on the order of about $1^{\circ}$, since the interfering effects of noise letters are greatly reduced if they lie outside this area. Hoffman (1975) suggested, from a recent study with this paradigm, that the spatially selective attentional processing occurred during the synthesis of recognizable forms from component features.

Questions about the specific process in which the attentional selection occurs, however, are raised by a number of experiments that have found no effects of spatial uncertainty on form perception. In tasks requiring the detection of a specific target or the discrimination between two alternative targets embedded within complex displays of other forms, Eriksen and Spencer (1969) and Shiffrin and Gardner (1972) have found that, with the exposure duration for each form held constant, accuracy was independent of the rate of presentation, whether the multiple forms were presented simultaneously or sequentially with no spatial uncertainty. Since the amount and rate of information acquired about a form at any given position was unaffected by the processing of forms at other positions, Shiffrin and Gardner (1972) concluded that "information enters the visual field and is perceptually processed 'without limitation' and without attentional control up to and including at least the level of letter recognition" (p. 82). Thus, prior knowledge about spatial position appears to have no effect on form recognition under these stimulus conditions. A critical condition for obtaining this effect seems to be that the targets must be held constant over trials and probably even over sessions, as recently indicated by a comprehensive series of studies by Shiffrin and Schneider (Note 1). In any event, the specific contribution of prior knowledge and selective attention to form perception is at present uncertain.

It might be supposed that spatial uncertainty would have no effect on the performance of a simple detection task not requiring identification or discrimination among alternative forms. This hypothesis is not confirmed, however. Pertinent evidence was recently reported by Posner, Nissen, and Ogden (Note 2), who found that the reaction time (RT) for detecting a light onset at an expected position was facilitated while the RT to a light at an unexpected position was inhibited relative to a neutral condition in which stimuli at both positions were equally likely. These effects were shown to occur without overt eye movements. Similarly, Cohn and Lasley (1974) demonstrated clear decrements in both $d^{\prime}$ and in the slope of the ROC for detecting luminance increments under conditions of spatial uncertainty. Moreover, these decrements were close to those predicted from signal detection theory to result from uncertainty about signal parameters (see Cohn, Thibos, \& Kleinstein, 1974; Nolte \& Jaarsma, 1967; Peterson, Birdsall, \& Fox, 1954). The basic idea is that when the signal is one of $n$ orthogonal alternatives, as when a light is presented at one of $\mathrm{n}$ equally likely positions, then the discriminant function for distinguishing signal + noise from noise alone is a vector in an n-dimensional space where it is only partially correlated with any one of the vectors corresponding to the individual signals. Thus, poorer detectability resulting from spatial uncertainty (or uncertainty about any other orthogonal stimulus parameters) is predicted for an ideal observer with no capacity limitations on sensory processes. So long as the noise process is uncorrelated among the alternative signals, lower detectability is an unavoidable effect from the statistical uncertainty of t the decision process, due to the increased number of opportunities for the noise alone to spuriously appear as an occurrence of the true signal. Therefore, a performance decrement resulting from an increased number of stimulus alternatives need imply neither a limitation on perceptual capacity nor the operation of an attentional selection. 
Returning to the present study, involving the detection of targets in random noise, it may be seen that a decline in detectability should be expected to occur with increased target positions, set size, because the random noise is generated so as to be uncorrelated between separate spatial positions. Even though the forced-choice detection task requires only a decision about whether a target form occurred in the first or second of two patterns, and not identification of the specific target, uncertainty about which of the alternative targets occurred should nevertheless affect the accuracy of the decisions. That is, the two patterns displayed on each trial need to be compared at each of the set of alternative positions in order to decide which is most likely to contain the target form. One pattern might seem to contain a target in one position, while the other pattern might also seem to contain a target in another position. As the number of alternative positions is increased, so too does the uncertainty about the relevant decision criterion, irrespective of any decrement in the visibility of the form per se. Thus, if the visual process for an individual target is modified by prior knowledge of the set of alternative targets, then the performance decrement with increased set size should exceed the decrement that is predicted to result from the increased uncertainty of the decision process. Otherwise, the decline in performance would more parsimoniously be attributed to the decision process rather than to visual perception per se.

In order to evaluate the obtained data against these predictable effects on the decision process, a simple model was constructed from just two assumptions. First, the observer was assumed to compare all of the alternative target positions in both patterns and to choose the pattern which contained the best approximation to a straight line in the set of alternative target forms. As the number of potential target positions increased, there was a corresponding lower likelihood that the true target would emerge from the set of competing alternatives.

Second, the process creating the "noise" pattern of camouflaging dots was assumed to be random, with all of the alternative positions varying independently with the same distribution. Thus, the general scheme is a signal detection model in which the signal is added to one of a set of independently and randomly varying "noisy" alternatives. If there are $n$ alternative positions in each pattern, then the total number of competing alternative postions is $2 n$. If $P_{n}$ represents the predicted percentage of correct responses with $\mathrm{n}$ alternative positions in each pattern, and if $P_{1}$ represents the probability that the target plus noise has a higher value than the pattern of noise at any given single alternative position, then it can be found from elementary probability theory (see Appendix) that the predicted per- centage of correct responses is given by the following formula:

$$
P_{n}=\frac{(n-1)-(n-2) P_{1}}{(2 n-1)-(2 n-2) P_{1}}
$$

If an attentional process, or some other perceptual contribution from the subject's prior knowledge of the set of alternative forms, were operating in the present experiment, then it should produce a larger effect of set size (n) than is predicted by Equation 1 . As we shall see, the close fit between the data and the predictions supports a decisional, rather than an attentional, interpretation.

\section{METHOD}

\section{Subjects}

Four undergraduate students at The University of Michigan were used as subjects. Each was paid a fixed stipend for a daily 1-h session, and none reported any evidence of visual abnormalities, although some wore corrective lenses. All subjects became highly trained and had served for at least one entire academic semester during which they participated in several similar experiments. They were therefore highly skilled at this type of task. Subjects were always familiarized with the alternative target forms prior to the collection of the experimental data.

\section{Apparatus}

Stimulus patterns were generated on the face of a cathode ray oscilloscope under the control of a small digital computer. Each pattern was composed of a dotted target form consisting of a single line of four dots embedded within 35 randomly positioned masking dots.

The target forms were prepared in advance by the experimenters and then stored in the computer for recall as requested by the experimental control program. A random-number algorithm was used by this control program to counterbalance the number and order of the targets to be presented within each subject's hourly session. The pattern of random masking dots was recalculated after each trial so that a new mask was used in each trial. The computer also controlled all response acquisition and data analysis procedures.

The subject viewed the CRT screen from a distance of $33 \mathrm{~cm}$. The viewing region on the face of the oscilloscope on which the random masking dots were presented was $5.6^{\circ} \times 5.6^{\circ}$, while stimulus lines were smaller, subtending an angle of only $1.88^{\circ}$. The luminance of each plotted dot was about $0.1 \mathrm{~cd} / \mathrm{m}^{2}$. The compartment in which the study was run was otherwise dark and soundproofed.

The subject's head was constrained in a fixed viewing position by a forehead switch. Unless this forehead switch contact was closed, two hand-held response switches were inactive and no responses could be entered into the computer.

Stimuli were plotted using an intensification pulse with a dura-, tion of $8 \mu \mathrm{sec}$ to the $z$ axis input of the oscilloscope. However, the duration of each dot was determined more by the persistence of the P-15 phosphor of the oscilloscope cathode ray tube than by this electronic pulse. According to the manufacturer's specifications, the P-15 phosphor has a persistence such that the light output is reduced to $0.1 \%$ of its initial brightness after $50 \mu \mathrm{sec}$. The apparent persistence of this briefly illuminated spot, of course, is elongated by the properties of the observer's visual system. However, the total physical duration of each pattern was about $1.0 \mathrm{msec}$. 


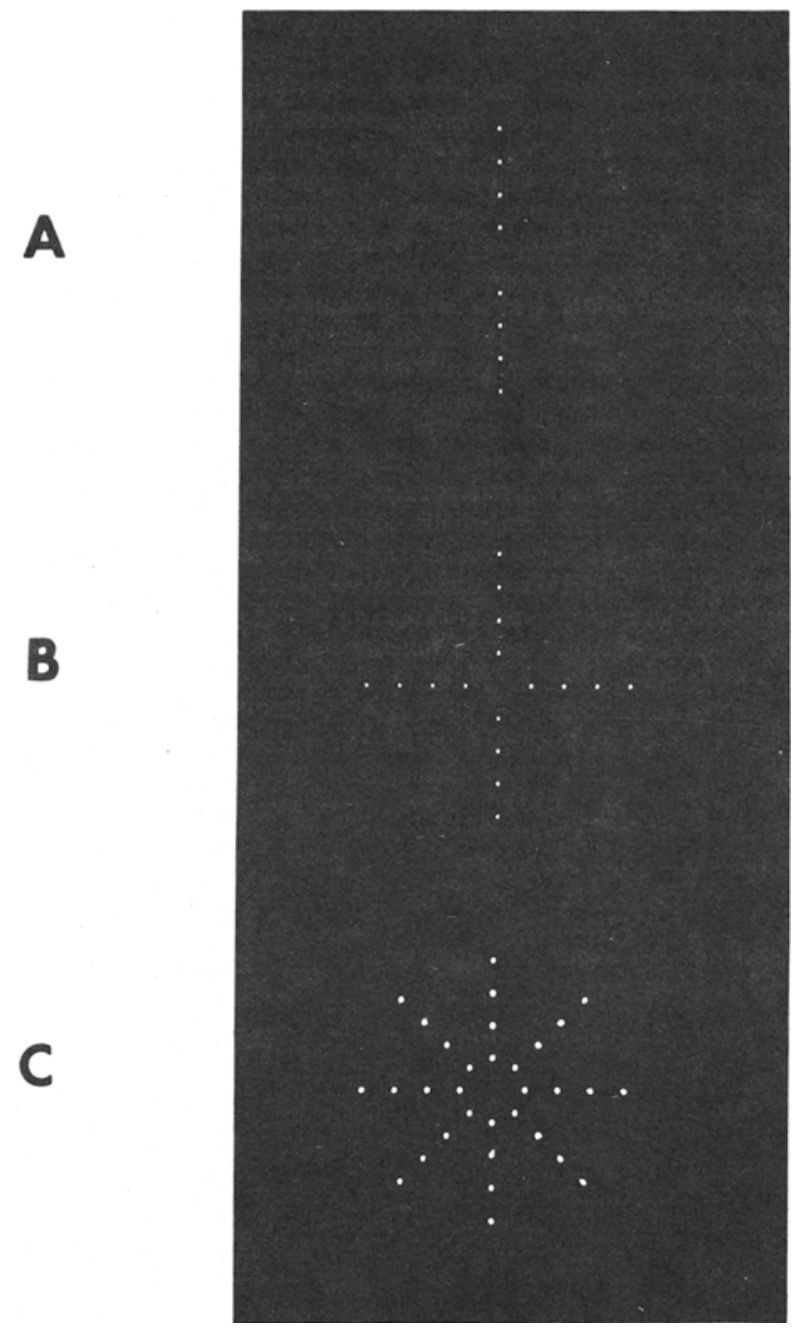

Figure 1. The three sets of target lines used in the experiment. Only one of the four-dot lines of each set was presented in the random noise in each trial.

\section{Procedure}

The experimental procedure was a two-alternative, forced-choice paradigm in which the subject was required to specify (by depressing one or the other of the two hand-held switches) which of two sequentially presented patterns (separated by $1.0 \mathrm{sec}$ ) contained one of the target lines in addition to the randomly positioned dotted visual masking dots common to both patterns. The nontarget pattern contained, in addition to the common masking dot pattern, a number of randomly placed dots equal to the number in the target line scattered over the region in which the target lines were placed to equate overall brightness and dot numerosity.

Following the depression of either one of the response switches, the subject received information concerning the correctness or incorrectness of his choice by means of a plus or a minus sign plotted on the oscilloscope screen. This feedback signal, which lasted for $.5 \mathrm{sec}$, also served as a fixation cue; there was no other fixation cue, other than the pattern itself, present in the intertrial or interstimulus intervals. The depression of the response switch and the presentation of the feedback signal was followed automatically by the presentation of the two patterns of the next trial. Pacing, therefore, was a function of the rate at which the subject responded.

All performance was measured as the percentage of the total number of presentations for which the target was correctly detected. A score of $50 \%$ represents chance performance in this two-alternative task. These data were accumulated for each subject and punched on a paper tape at the end of each session. At the end of the day, a secondary data analysis program was used to summarize the results from all subjects.

The target form used in each trial consisted of a single dotted radial line selected from among three sets as shown in Figure 1. One set consisted of only two vertical lines. The second set consisted of four lines, two horizontal and two vertical, while the third consisted of eight lines spaced $45^{\circ}$ apart. The computer program randomly selected a single line for each trial from the set being used on each day.

The three sets of targets were presented on 6 successive days. On the first 3 days, set sizes 2,4 , and 8 were presented in this ascending order. On the second 3 days, the three set sizes were presented in the reverse descending order to counterbalance a sequence effect. Data from corresponding pairs of sessions were then pooled to reduce the possibility of any sequence effect biasing the results.

Since each tabulated stimulus was presented a variable number of times depending upon set size, the data plotted in Figure 2 varies from point to point. For set size 2 , each data point represents a sample of approximately 6,400 trials; for set size 4, 3,200 trials; and for set size 8, 1,600 trials (combining two sessions for each of four subjects).

\section{RESULTS}

The data were the percentages of correct responses to the two line positions (the two verticals) that appeared in all three set sizes. The data from the other positions in the sets of four and eight alternatives were not analyzed, since they were not relevant to the present experimental question.

Figure 2 shows the observer and predicted percentages of correct responses $\left(P_{n}\right)$ as a function of size of the set of target forms. The predicted func-

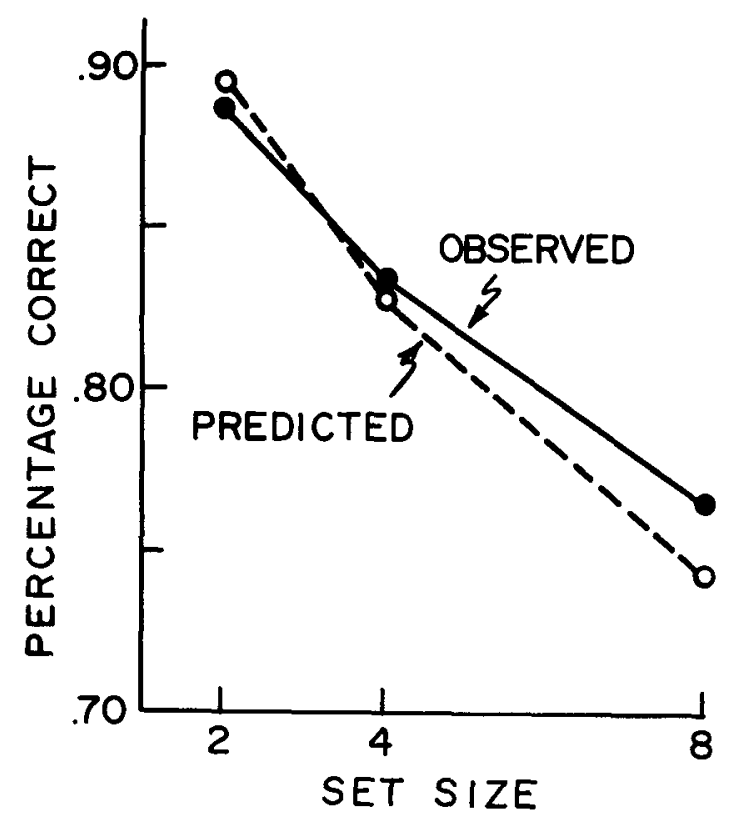

Figure 2. The obtained and predicted percentages of correct responses for detecting the two vertical target lines as a function of set size. 
tion was obtained by using Equation 1 to estimate the parameter $P_{1}$ for each session; the 24 estimates were averaged, and this average value was then used in Equation 1 to obtain the predicted values shown in Figure 2. As may be seen, the observed effect is large but does not exceed the predicted effect of set size on detection accuracy. The largest discrepancy is for the set size 8 condition, where the predicted accuracy is about $2 \%$ below the observed value.

A statistical comparison of the observed and predicted values was made by testing for the hypothesized invariance of the parameter $P_{1}$ for various set sizes. That is, if set size has no effect on the perceptual information but only on the decision criterion for selecting a response, then estimates of $P_{1}$, the hypothetical probability of a correct response with a set size of 1 , should be invariant across the experimental conditions. The average values of $P_{1}$ estimated for set sizes 2,4 , and 8 , respectively, were $.935, .943$, and .946 . The reliability of the differences between these estimates was tested by a Friedman two-way analysis of variance by ranks (Siegel, 1956) on the rank-ordering of the three estimates across the eight replications of the experiment-twice for each of four subjects. The ranking of these values was inconsistent, so that the Friedman statistic did not approach significance $\left(\chi_{\mathrm{r}}^{2}=2.25\right.$, $\mathrm{df}=2, \mathrm{p}=.355$ ). Moreover, the three estimates were typically quite similar; the largest difference between any two values in one of the eight replications was less than $4 \%$.

\section{DISCUSSION}

The results of this experiment do not indicate that prior knowledge about the position and orientation of a line facilitates detection of the line in random visual noise. Although increases in the size of the set of target forms do lead to decrements in accuracy of the detection responses, these decrements do not exceed what would be expected if the random noise that hides the target varied independently at all of the possible positions. Detection accuracy, according to this analysis, drops simply because there are more opportunities at the larger number of alternative positions for the noise to be confused with the target, not because the target is itself any less visible. Such spatial independence in the random pattern of noise dots, it may be noted, is a basic assumption of the autocorrelation theory that led to this experiment.

These results are thus compatible with the autocorrelation theory: Form detectability seems to be determined by the structural characteristics of the stimulus pattern, irrespective of the observer's prior knowledge about the form.

How general are these results? Of course, they are subject to the usual qualifiers about the specific conditions examined in this experiment-the limited retinal area, the limited sizes of the sets of alternative forms, the limited variation in stimulus characteristics, a detection rather than a recognition task, etc. Presumably, prior knowledge-selective attention, expectancy, etc. - can facilitate some aspects of form perception under some conditions, and it is important to understand how and when such effects occur. Although the present experiment provides no direct information about the nature of the boundary conditions for the null result that was obtained, comparison with other experiments may help to place this result in perspective.

First, the intent of the present experiment was to examine sets of forms in which the alternatives were distinctly different from each other, on the assumption that prior knowledge of the alternatives would be most likely to enable attention to selected features under such conditions. The failure to find evidence for such selective processing under the present conditions would therefore suggest that prior knowledge is not likely to facilitate the perception of forms varying on more subtle aspects of geometry-e.g., as in sets of alphanumeric characters. However, since the most directly relevant experiment, on the recognition of various sized sets of alphanumeric characters in random dotted noise, has not been conducted, its result remains an untested speculation.

Numerous other related experiments, though, have been conducted-e.g., on the speed of recognizing alphanumeric characters as a function of set size. The general result of the latter class of experiments is well known and often replicated: The reaction time $(\mathrm{RT})$ for recognizing that a presented form is a member of the target set increases as an approximately linear function of set size (e.g., Sternberg, 1966). This result is qualitatively similar to the result of the present experiment: In the former case, increased set size leads to longer RTs at a presumably constant accuracy criterion; in the present case, increased set size leads to decreased accuracy at a presumably constant visual response latency. Both effects imply that with increased set size the discrimination task becomes more difficult and the stimulus presentation acquires control over the response selection at a slower rate.

Nevertheless, the two experimental results have led to different theoretical interpretations: The effect of set size on RT is usually interpreted as an index of the search rate with which the stimulus is compared to the set of alternatives in memory, while the effect of set size on detection accuracy in the present experiment is interpreted as an inherent aspect of the decision process, involving no limitation on information processing. The difference between these interpretations does not derive from empirical operations or results, but from differing initial representations of the stimulus information. From the standpoint of information processing, stimulus in- 
formation is usually specified by the particular stimulus presented on each trial. When a given stimulus and its response alternatives are constant across conditions, then any effect from the subject's prior knowledge of the alternative stimuli would seem to result from characteristics of the subject's processing of the stimulus rather than from characteristics of the objective stimulus and response. On the other hand, from the standpoint of a decisiontheoretic analysis of discrimination between noiseperturbed alternatives, stimulus information is defined relatively rather than absolutely-on the comparative relations that differentiate among the alternatives rather than on the characteristics of a given individual stimulus. Changing the set of alternatives also changes the relevant characteristics of an individual stimulus. Increasing the set size increases the observer's uncertainty about the relevant stimulus features and accordingly increases the number of opportunities for noise to be mistaken for a target.

These detrimental effects of set size on the selection of a decision criterion are easy to understand in the present experiment where the noise occurs independently at each of the potential target positions, but the effect is perhaps less obviously applicable to experiments on the speed of recognition of easily detectable stimuli. Nevertheless, the isomorphism between the two tasks suggests that analogous processing problems may be involved in the performance of both tasks. Perhaps the speed of recognition is slowed by an increase in set size not because the presented stimulus is processed at a slower rate, but because the response criterion must be based on more stimulus features, with a corresponding reduction of the signal/noise ratio for detecting the true target after a given amount of processing; longer processing would therefore be required to bring the signal/noise ratio to a given level of accuracy. Anderson's (1973) model seems to be one of the few theoretical acknowledgments that set size should have this predictable effect on the speed of recognition.

If form detection and recognition may be understood as involving something akin to a crosscorrelation between the target and the stimulus pattern, then perhaps it should not be surprising that Eriksen and Spencer (1969) and Shiffrin and Gardner (1972) found no effect from the rate of stimulus presentation: When both the target and the number of noise items are held constant across conditions, then spatial uncertainty should have no effect on the total number of discriminations required in their task, as was found. Increasing the number of noise items, however, should have produced a decrement in target detection just as in the present experiment, and such an effect was indeed obtained by Eriksen and Spencer (1969). What is suprising, however, is Shiffrin and Schneider's (Note 1) finding that after extended practice with a given set of targets, set size had almost no effect on the speed and accuracy of recognition. Perhaps with sufficient practice the stimulus features become perceptually correlated so that the minimal number of necessary distinctive features becomes independent of the size of the set of target forms; or perhaps several separate response criteria can eventually be employed simultaneously. At any rate, the result does not seem readily interpretable in terms of the present theoretical position.

The results of this experiment should not be understood as an indication that selective attention to spatial positions cannot or does not occur under other conditions. Such selective processing seems clearly demonstrated by the work of Eriksen and his colleagues (e.g., Colegate et al., 1973; Eriksen \& Hoffman, 1972, 1973; Hoffman, 1975). Apparently, the noise processes that operate in those experiments involve response confusions among forms occurring within about $1^{\circ}$ of visual angle (Eriksen \& Hoffman, 1972, 1973).

In conclusion, the principal value of the present experiment is simply the illustration of how prior knowledge can facilitate the decision in detection and recognition tasks. If the effect of set size can be attributed to uncertainty about target parameters, and is therefore predictable for an ideal observer, then it is unnecessary and perhaps misleading to propose limitations on information processing as an explanation for the effect. This interpretation represents an application of the theorem that the imperfection of a real observer can often be simulated by an ideal observer with statistical uncertainty about the target parameters (see Tanner \& Birdsall, 1958). The theoretical implication is that this aspect of the decision process may be sufficient to account for a major portion of observed effects of prior knowledge on detection and recognition.

\section{APPENDIX}

To obtain a prediction for the effect of set size on the accuracy of detection, it is necessary first to represent the relation between the probability of locating the target form in a pattern of noise and the observed accuracy of choosing the correct response that indicates which of the two patterns contained the target form. The correct response might be chosen either because the target form was correctly located in the pattern or because one of the other alternative target positions in the same pattern falsely appeared to contain the form. The probability of a correct response is thus the probability of the union of two sets of events. By elementary laws of probability theory, the probability of the union of sets is equal to the complement of the probability of the intersection of the complements of the sets. That is, if $L$ represents the correct location of the target form and $F$ represents the false location of the 
form in one of the other positions in the same pattern, then the predicted proportion of correct responses, $P$, is given by

$$
\begin{aligned}
\mathbf{P} & =\mathrm{p}(\mathrm{L} \text { or } \mathrm{F}) \\
& =1-\mathrm{p}(\text { not-L and not-F })
\end{aligned}
$$

When the events not- $L$ and not-F can be assumed to be stochastically independent, as is appropriate when errors are presumed to result from a randomly varying noise process that is independent in separate positions, then

$$
\begin{aligned}
P & =1-p(\text { not }-L) \cdot p(\text { not-F }) \\
& =1-[1-p(L)] \cdot[1-p(F)] .
\end{aligned}
$$

Next, consider the detectability of a target embedded within a set of independently and randomly varying "noisy" alternatives. If $P_{1}$ were the probability of a correct response when the form was presented at a single position in one of two alternative patterns, then $1-P_{1}$ would be the probability that the noisy alternative would be mistakenly confused for the target. If there are two alternative target positions in each pattern, then the target appears in one of four possible locations and must be discriminated from three noisy alternatives. The probability of detecting the form in the total set of four alternatives would then be

$$
p(L)=\frac{P_{1}}{P_{1}+3\left(1-P_{1}\right)}
$$

In general, if there are $\mathrm{n}$ alternative target positions in each of two patterns, then the probability of locating the form in the correct position of the correct pattern would be given by

$$
p(L)=\frac{P_{1}}{P_{1}+(2 n-1)\left(1-P_{1}\right)}
$$

Finally, consider how $p(F)$, the probability of a false location of the form in one of the other positions in the correct pattern, varies as a function of $n$. If all $(2 n-1)$ of the positions in both patterns are equally likely to be confused with the true target, then the probability of a false location occurring at one of the $n-1$ remaining positions in the correct pattern is given by

$$
p(F)=\frac{n-1}{2 n-1}
$$

Substituting from Equations A-2 and A-3 into Equation A-1 yields:

$$
\begin{aligned}
P_{n} & =1-\left[1-\left(\frac{P_{1}}{P_{1}+(2 n-1)\left(1-P_{1}\right)}\right)\right]\left[1-\frac{n-1}{2 n-1}\right] \\
& =1-\left\lfloor\frac{(2 n-1)\left(1-P_{1}\right)}{P_{1}+(2 n-1)\left(1-P_{1}\right)}\right\rfloor\left[\frac{n}{(2 n-1)}\right\rfloor
\end{aligned}
$$

$$
\begin{aligned}
& =1-\frac{n\left(1-P_{1}\right)}{P_{1}+(2 n-1)\left(1-P_{1}\right)} \\
& =\frac{(n-1)-(n-2) P_{1}}{(2 n-1)-(2 n-2) P_{1}}
\end{aligned}
$$

Solving for $P_{1}$ in this equation, the parameter $P_{1}$ may be estimated from the obtained percentage of correct responses by the formula

$$
P_{1}=\frac{(n-1)-(2 n-1) P_{n}}{(n-2)-(2 n-2) P_{n}}
$$

The principal assumptions in this derivation are that all of the $(2 n-1)$ positions containing only random noise are independently and, on the average, equally confusable with the true target. Detection accuracy is predicted to vary with set size because the observer's knowledge of the set of alternative targets should determine the decision criteria for choosing the response on a given trial. The effect is predicted for an ideal observer whose sensory processes are unaffected by set size.

Similar predictions can be derived from the stronger assumption associated with signal detection theory that the noise is normally distributed with the same mean and variance independently at each location (see Cohn \& Lasley, 1974; Nolte \& Jaarsma, 1967; Peterson, Birdsall, \& Fox, 1954). The prediction given in Equation 1 is more general in that it assumes nothing about the form of the probability density function of the noise and nothing about the psychophysical function relating detectability to signal/ noise ratio. Equation 1 is less general, however, in that it applies only to the forced-choice task and makes no prediction about the ROC curve for yes/no tasks. A different but related formulation based on communication theory is also given by Anderson (1973).

\section{REFERENCE NOTES}

1. Shiffrin, R. M., \& Schneider, W. A two-process model for attention and search. Indiana Cognitive/Mathematical Psychology Conference, Bloomington, A pril 1976.

2. Posner, M. I., Nissen, M. J., \& Ogden, W. Attention to a position in space. Paper given at the Annual Meeting of the Psychonomics Society, Denver, November 1975.

\section{REFERENCES}

ANDERson, J. A. A theory for the recognition of items from short memorized lists. Psychological Review, 1973, 80, 417-438.

CoHn, T. E., \& LasLEY, D. J. Detectability of a luminance increment: Effect of spatial uncertainty. Journal of the Optical Society of America, 1974, 64, 1715-1719.

Cohn, T. E., Thibos, L. N.. \& KLeinstein, R. N. Detectability of a luminance increment. Journal of the Optical Society of America, 1974, 64, 1321-1327.

Colegate, R. L., Hoffman, J. E., \& Erixsen, C. W. Selective encoding from multielement visual displays. Perception \& Psychophysics, 1973, 14, 217-224.

ERIKsen, C. W., \& Hoffman, J. E. Temporal and spatial characteristics of selective encoding from visual displays. Perception $\&$ Psychophysics, 1972, 12, 201-204. 
Eruksen, C. W., \& Hoffman, J. E. The extent of processing of noise elements during selective encoding from visual displays. Perception \& Psychophysics, 1973, 14, 155-160.

ERIKSEN, C. W., \& SPENCER, T. Rate of information processing in visual perception: Some results and methodological considerations. Joumal of Experimental Psychology, 1969, 79, No.2, Part 2.

Hoffman, J. E. Hierarchical stages in the processing of visual information. Perception \& Psychophysics, 1975, 18, 348-354.

Nolte, L. W., \& JAArsma, D. More on the detection of one of $M$ orthogonal signals. Joumal of the Acoustical Society of America, $1967,41,497.505$.

Peterson, W. W., Birdsall. T. G., \& Fox, W. C. The theory of signal detectability. IRE Transactions on Information Theory, $1954,4,171-212$.

SHIFFrin, R. M., \& GARDNER, G. T. Visual processing capacity and attentional control. Journal of Experimental Psychology,
1972, 93, 72-82.

SIEGEL, S. Nonparametric statistics for the behavioral sciences. New York: McGraw-Hill, 1956.

Sternberg, S. High-speed scanning in human memory. Science, $1966,153,652-654$.

TANNER, W. P., JR., \& Birdsall, T. G. Definitions of d' and $\eta$ as psychophysical measures. Journal of the Acoustical Society of America, 1958, 30, 922-928. Also in J. A. Swets (Ed.), Signal detection and recognition by human observers. New York: Wiley, 1964.

UTTAL, W. R. An autocorrelation theory of form detection. Hillsdale, N.J: Lawrence Erlbaum Associates, 1975.

(Received for publication March 24, 1976; revision accepted August 3, 1976.) 